\title{
The Thalamus: A Small but Precious Window on $\tau$-Related Neurodegeneration?
}

$\mathbf{P}$ rogressive supranuclear palsy (PSP) and corticobasal degeneration (CBD) are pathologically distinct causes of progressive atypical parkinsonism, poorly responsive or not responsive to levodopa. ${ }^{1}$ Both diseases are characterized by hyperphosphorylated $\tau$ aggregates in the brain, accompanied by neuronal loss and gliosis in a characteristic distribution. ${ }^{1}$ The large majority of patients with PSP present with Richardson syndrome, also known as PSP syndrome, ${ }^{2}$ characterized by postural instability, leading to backward falls within the first year of symptom onset; axial rigidity; progressive vertical ophthalmoplegia; dementia; and personality changes. CBD pathology can cause multiple different neurologic syndromes, including corticobasal syndrome, PSP syndrome, frontotemporal dementia, or primary progressive aphasia. Corticobasal syndrome includes a mixed movement disorder (eg, levodopaunresponsive rigidity associated with apraxia, dystonia, myoclonus, and alien limb) and impaired cognition. ${ }^{3}$ An early and accurate diagnosis of tauopathies in vivo is becoming increasingly important with the advent of clinical trials of drugs aimed at modifying the underlying $\tau$ pathology. However, outside specialized centers and especially in the early stage of the diseases, differential diagnosis among atypical parkinsonian syndromes and with Parkinson disease (PD) is challenging because of the similarity of symptoms and lack of preclinical markers of the disease.

The thalamus is a major conduit for the bidirectional flow of neuronal signals between cortical and subcortical regions and links different cortical regions via transthalamic pathways. It comprises numerous nuclei, which receive a specific set of afferent connections and project primarily within the confines of specific cortical areas. Thalamic degeneration is known to occur in PSP and $\mathrm{CBD}$, though the topographic distribution of thalamic lesions differs between the 2 pathologies. ${ }^{4-6}$ A recent $\left[{ }^{18} \mathrm{~F}\right] 2-(1-\{6-[2-$ [fluorine-18]fluoroethyl)(methyl)amino]-2-naphthyl\}-ethylidene)malononitrile positron-emission tomography study showed the presence of $\tau$ fibrillar aggregates in the thalamus of patients with PSP syndrome. ${ }^{7}$ Thalamic damage occurs also in patients with $\mathrm{PD}$; however, it is less severe and is likely to occur later in the course of the disease compared with PSP and CBD. ${ }^{5,8}$ Thus, measures of thalamic involvement may be useful in the differential diagnosis of these neurodegenerative conditions.
It is technically very difficult to assess accurately the thalamic subnuclei in vivo by using imaging technology. Previous studies of PD and atypical parkinsonisms demonstrated thalamic abnormalities by using region-of-interest or voxelwise approaches. ${ }^{9-13}$ MR imaging-based analysis of the shape of the subcortical structures provides useful pieces of information about the location and pattern of structural abnormalities associated with these pathologic conditions. Using such an approach, several studies reported regional thalamic damage in patients with schizophrenia, obsessive-compulsive disorders, Alzheimer disease, and Tourette syndrome. ${ }^{14-16}$ However, only a few MR imaging studies so far have used this approach in patients with parkinsonian syndromes and have focused their attention on the differences between disease groups and healthy controls, and not among different patient groups. ${ }^{17,18}$

Against this background, the preliminary study by Hess et $\mathrm{al}^{19}$ published in the present issue of the American Journal of Neuroradiology has investigated thalamic degeneration in a small sample of patients with PSP syndrome, corticobasal syndrome, and PD by using an approach that combines shape analysis and diffusion tensor imaging. The aim of the study ${ }^{19}$ was to evaluate whether the regional patterns of thalamic atrophy and microstructural damage differ in patients with atypical parkinsonian syndromes compared with those with idiopathic PD. By contrasting the volumes of the thalamus among different patient groups, the authors demonstrated that this structure undergoes more atrophy in patients with PSP syndrome and corticobasal syndrome than in those with PD. Shape analysis also provided insight into the topography of thalamic tissue loss, which was driven by a diminished size of the ventral anterior and ventral lateral portions of the structure. The DTI-based analysis showed that mean apparent diffusion coefficient values were higher in patients with PSP syndrome compared with those with PD and control groups, and shape analysis revealed that microstructural abnormalities were greater within the motor regions of the thalamus in both PSP syndrome and corticobasal syndrome groups relative to PD and controls.

Overall this article provides new evidence for thalamic damage occurring in atypical parkinsonisms, highlighting the preferential 
involvement of lateral (motor) nuclei that are, instead, relatively spared in patients with PD. The selective involvement of thalamic motor nuclei and, as a consequence, of motor thalamocortical circuits in PSP syndrome and corticobasal syndrome may be interpreted as a regionally specific vulnerability to $\tau$-related neurodegeneration. Therefore, in the near future, structural and diffusion tensor MR imaging is likely to provide useful markers to distinguish patients with atypical parkinsonisms not only from healthy individuals but also from patients with $\mathrm{PD}$, which is the actual clinical dilemma.

The study by Hess et $\mathrm{al}^{19}$ is hampered by a number of limitations, as acknowledged by the authors. The small number of subjects and the inclusion of cases not pathologically confirmed limit the generalizability of their findings and transferability to clinical practice. In addition, thalamic networks are involved in motor behavior, but they are also related to emotional, motivational, associative, and cognitive abilities. As a consequence, future studies should explore the relationship between regional thalamic damage and cognitive and behavioral findings in subjects with PSP syndrome and corticobasal syndrome.

Although the approach of this study requires further work to assess its consistency and reproducibility, the results reported by Hess et $\mathrm{al}^{19}$ provide a solid justification to use a combination of shape analysis and structural and/or functional connectivity to study the pattern of thalamic degeneration in larger populations with pathologically proved PSP syndrome and corticobasal syndrome. Such studies could enable researchers to improve knowledge on these pathologic processes and overhaul the way we approach drug discovery for these devastating conditions.

\section{REFERENCES}

1. Dickson DW, Kouri N, Murray ME, et al. Neuropathology of frontotemporal lobar degeneration-tau (FTLD-tau). J Mol Neurosci 2011;45:384-89

2. Litvan I, Agid Y, Calne D, et al. Clinical research criteria for the diagnosis of progressive supranuclear palsy (Steele-RichardsonOlszewski syndrome): report of the NINDS-SPSP international workshop. Neurology 1996;47:1-9

3. Armstrong MJ, Litvan I, Lang AE, et al. Criteria for the diagnosis of corticobasal degeneration. Neurology 2013;80:496-503

4. Hattori M, Hashizume $Y$, Yoshida M, et al. Distribution of astrocytic plaques in the corticobasal degeneration brain and comparison with tuft-shaped astrocytes in the progressive supranuclear palsy brain. Acta Neuropathol 2003;106:143-49

5. Halliday GM, Macdonald V, Henderson JM. A comparison of degeneration in motor thalamus and cortex between progressive supranuclear palsy and Parkinson's disease. Brain 2005;128:2272-80

6. Ling H, O'Sullivan SS, Holton JL, et al. Does corticobasal degenera- tion exist? A clinicopathological re-evaluation. Brain 2010;133: 2045-57

7. Kepe $\mathrm{V}$, Bordelon $\mathrm{Y}$, Boxer A, et al. PET imaging of neuropathology in tauopathies: progressive supranuclear palsy. J Alzheimers Dis 2013;36:145-53

8. Henderson JM, Carpenter K, Cartwright H, et al. Loss of thalamic intralaminar nuclei in progressive supranuclear palsy and Parkinson's disease: clinical and therapeutic implications. Brain 2000; 123(pt 7):1410-21

9. Erbetta A, Mandelli ML, Savoiardo M, et al. Diffusion tensor imaging shows different topographic involvement of the thalamus in progressive supranuclear palsy and corticobasal degeneration. AJNR Am J Neuroradiol 2009;30:1482-87

10. Agosta F, Pievani M, Svetel M, et al. Diffusion tensor MRI contributes to differentiate Richardson's syndrome from PSP-parkinsonism. Neurobiol Aging 2012;33:2817-26

11. Boxer AL, Geschwind MD, Belfor N, et al. Patterns of brain atrophy that differentiate corticobasal degeneration syndrome from progressive supranuclear palsy. Arch Neurol 2006;63:81-86

12. Padovani A, Borroni B, Brambati SM, et al. Diffusion tensor imaging and voxel based morphometry study in early progressive supranuclear palsy. J Neurol Neurosurg Psychiatry 2006;77:457-63

13. Messina D, Cerasa A, Condino F, et al. Patterns of brain atrophy in Parkinson's disease, progressive supranuclear palsy and multiple system atrophy. Parkinsonism Relat Disord 2011;17:172-76

14. Kang DH, Kim SH, Kim CW, et al. Thalamus surface shape deformity in obsessive-compulsive disorder and schizophrenia. Neuroreport 2008;19:609-13

15. Zarei M, Patenaude B, Damoiseaux J, et al. Combining shape and connectivity analysis: an MRI study of thalamic degeneration in Alzheimer's disease. Neuroimage 2010;49:1-8

16. Wang L, Lee DY, Bailey E, et al. Validity of large-deformation high dimensional brain mapping of the basal ganglia in adults with Tourette syndrome. Psychiatry Res 2007;154:181-90

17. McKeown MJ, Uthama A, Abugharbieh R, et al. Shape (but not volume) changes in the thalami in Parkinson disease. BMC Neurol 2008;8:8

18. Saini J, Bagepally BS, Sandhya M, et al. Subcortical structures in progressive supranuclear palsy: vertex-based analysis. Eur J Neurol 2013;20:493-501

19. Hess CP, Christine CW, Apple AC, et al. Changes in the thalamus in atypical parkinsonism detected using shape analysis and diffusion tensor imaging. AJNR Am J Neuroradiol 2014;35:897-903

M. Filippi

F. Agosta

F. Caso

Neuroimaging Research Unit Institute of Experimental Neurology Division of Neuroscience

San Raffaele Scientific Institute Vita-Salute San Raffaele University Milan, Italy

http://dx.doi.org/10.3174/ajnr.A3930 